were the result of a deficiency of vitamin $D$. A possible mechanism for this is suggested by the significant association of hypocalcaemia and a raised liver phosphatase isoenzyme in the serum. Phenobarbitone and phenytoin can induce the liver enzymes responsible for the metabolism of many steroid compounds-for example, cortisol (Kuntzman, 1969)-and therefore probably vitamin $\mathbf{D}$ metabolism might be similarly affected. The importance of the liver in vitamin $D_{3}$ metabolism was underlined by the demonstration in patients with cirrhosis of a prolonged plasma half-life of the vitamin and a reduced renal excretion of its glucuronide conjugates (Avioli, Lee, McDonald, Lund, and DeLuca, 1967). Induction of liver enzymes by anticonvulsant drugs would shorten the biological half-life and lead to a tissue deficiency of the vitamin despite an adequate dietary intake. Subsequent osteomalacia would lead to an increase of bone alkaline phosphatase in the serum. This hypothesis is discussed further by Dent et al., (1970), and is at present being tested in rats by one of us (D.J.F.R.). The results of an initial study showed that liver enzyme induction with phenobarbitone significantly protected rats against loss of weight, hypercalcaemia, and renal calcinosis produced by acute administration of a high dose of calciferol. Further experiments are being performed to confirm these observations and to examine the other anticonvulsant drugs for a similar protective effect.

We would like to thank Dr. John Laidlaw, senior physician at the Chalfont Centre for Epilepsy, for allowing us to investigate patients under his care; the staff at Chalfont and University College Hospital who acted as controls; Professor C. E. Dent and Dr. T. C. B. Stamp for advice and discussion; the chemical pathology department at U.C.H. for serum protein estimations; and Mr. D. S. L. Lloyd for compuxing advice and programmes.

\section{REFERENCES}

Avioli, L. V., Lee, S. W., McDonald, J. E., Lund, J., and DeLuca, H. F. (1967). Fournal of Clinical Investigation, 46, 983.

Canapa-Anson, R., and Rowe, D. J. F. (1970). Fournal of Clinical Pathology. In press.

Dent, C. E. (1962). British Medical fournal, 2, 1419.

Dent, C. E., Richens, A., Rowe, D. J. F., and Stamp, T. C. B. (1970). British Medical Fournal, 4, 69.

Kruse, R. (1968). Monatsschrift für Kinderheilkunde, 116, 378.

Kuntzman, R. (1969). In Annual Review of Pharmacology, 9, 21

Rudra, M. N., De, S., and Rudra, M. S. (1969). New England Yourmal of Medicine, 280, 1242.

Vas, C. J., and Parsonage, M. J. (1967). Acta Neurologica Scandinavica, 43, Wright, J. A. (1965). Epilepsia, 6, 67.

\title{
Computer-held Clinical Record System-I, Description of System
}

\author{
L. J. OPIT, ${ }^{*}$ B.SC., F.R.C.S., F.R.A.C.S. ; F. J. WOODROFFE, $†$ M.B., B.S., M.R.C.P.
}

British Medical fournal, 1970, 4, 76-79

Cummary: An account of a computer system for clinical record, storage, and retrieval is presented. Some details of its design and implementation are described.

\section{Introduction}

During the past five years there has been an increasing volume of literature devoted to the role of the computer in clinical medicine. Despite this, papers reporting on details of design and problems of working, or experimental, medical record computing systems are scarce. In this paper we attempt to describe in some detail how one computer system, King's College Hospital real-time computer project (Phase I), works.

\section{Present System}

The present King's College Hospital system provides a facility for up to four doctors working independently either to (1) enter registration details of patients or (2) record details of a history, physical examination, diagnosis, vital signs, and progress notes. The project also enables the senior ward nursing staff to record certain nursing routines prescribed for the patient.

Because at the outset it was proposed to install this system in two medical wards we restricted ourselves to providing mainly for the sort of information likely to be needed on a general medical ward. The device which the doctor uses to record his findings is a visual display unit (Fig. 1). This is a small television-like display screen with an attached keyboard, and is kept on a mobile trolley in the ward.

- Senior Lecturer, King's College Hospital Computer Unit, London S.E.5. t Consultant Physician to the Forest Group of Hospitals. Present appointt Consultant Physician to the Forest Group of Hospitals. Present appointment: Senior Research
sity of Birmingham.
The information which the doctor records by using the visual display unit is filed away on a magnetic storage device, which in the King's system is an exchangeable disc. At the same time as the messages are filed to the disc they are copied on to two magnetic tapes. These tapes are kept for only 24 hours and provide a means of reconstructing the whole of the last 24-hour period up to the time of any breakdown. Every message about a patient is stored on an openended file on this disc, the patient's record being identified by name and hospital number. These messages are kept logically, in the order in which they arrive. As well as the capacity to record information about each patient the visual display unit may be used to inspect messages already existing in the patient's file. These messages are displayed on the visual display unit screen, one at a time, and by typing the number

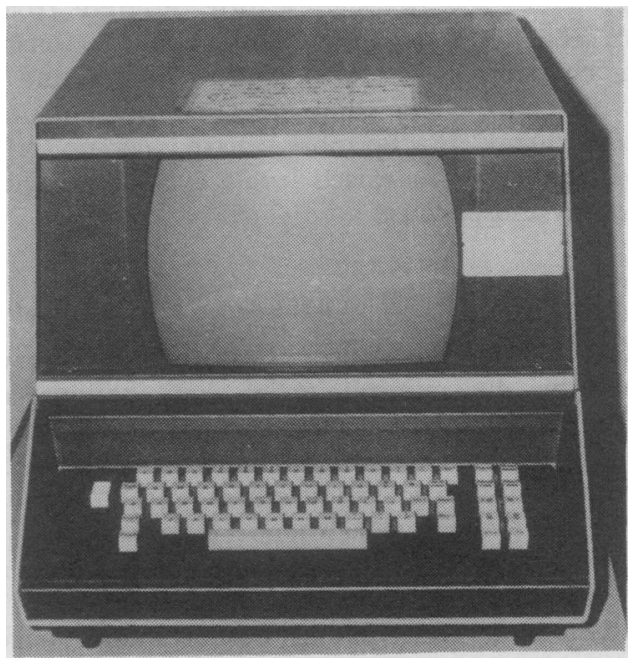

Fig. 1.-Visual display unit. 
1 or 2 on the keyboard of the instrument it is possible to flip visually backward or forward through the file. Moreover, a daily print out is provided by the computer system. This paper record is a print out of the contents of each patient's disc file. The details and format of these notes are presented below.

\section{Input}

It is now possible to indicate precisely how the patient's medical record is built up. The doctor selects numbered lines of text presented to him on the screen of the visual display unit. The required line is selected by typing the appropriate number and then pressing two other keys, which signify the completion of the choice and transmit it to the computer. This action causes the text to be kept and automatically presents the next appropriate screen.

\section{Displays}

These static pictures on the screen are referred to as displays. These displays are kept on another file and are automatically transmitted to the visual display unit via the central processor of the computer as the user performs certain actions on the keyboard of the visual display unit. The displays are interlocked in a complex way. For example, selecting a symptom, such as pain, from the screen by typing the appropriate number causes a display to appear which enables the doctor to choose to specify details of site, onset, or duration of pain, etc. The design of the display file was undertaken by a team of doctors and took some 30 manmonths in actual writing. This interlocking (and other features) is in fact specified in advance by the designers of the displays. The user does not need to know what to do next because the response of the system is automatically determined by the designer. The number of displays needed to cope with the requirements of the general medical ward was about 3,800 . These displays are on a special file, the contents of which may be amended, deleted, or added to, so that continuous modification of the system is possible. We have not yet had sufficient experience with the system to decide whether these displays will meet every situation or indeed how many of them are redundant.

An interesting sidelight thrown up in the design of the system has been the finding that some 8,000 different words have been used. A medical dictionary contains some 20,000 terms. Evidently, therefore, a doctor's working vocabulary may be a lot smaller than the total available.

A full account of the problems met in display design and which were imposed by machine programming and other restrictions occurring at King's College Hospital is presented in the following article. In certain circumstances the text is not stored, though some action is generated by the choice of a number. This occurs when, for example, the text provided is only to indicate the next action-for example, "End of Exam1nation of Heart." The text will also be suppressed from the patient's record when there is redundancy of certain words. Thus a choice of "ONSET" may lead to a display which has as its content "GRADUAL ONSET," "SUDDEN ONSET," etc. The first use of "ONSET" provides a guide for action but is redundant as text. These suppressions are specified in advance by the display designer, and the user has no knowledge of them.

\section{Types of Display}

Three functionally distinct types of display have been developed in this system, and the screen appearance of typical examples is presented as they would appear during recording. "p" Display

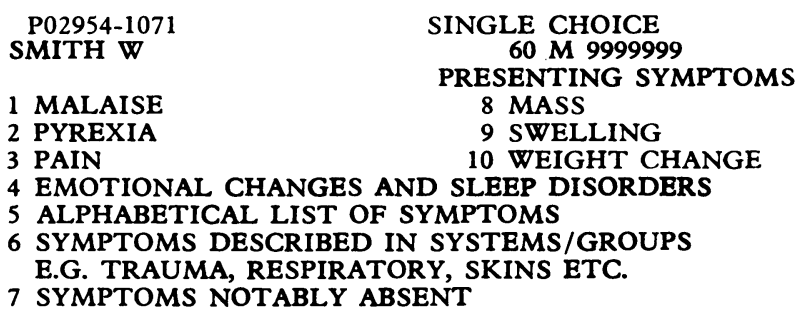

This type of display may occupy 10 lines of screen with up to 20 possible numbered choices; only one choice at a time may be exercised and each choice may lead to a different display.

“Q” Display
Q00222-0077
SMITH W
S0 M 99999999 CHOICES
1 TENDER
SUPERFICIAL VEINS 0
2 OVERLYING SKIN REDDENED
3 HARD
4 VARICOSE
5 DISTENDED
6 FLOW REVERSED

These are similar to "P" displays except that any number of choices may be exercised from each screen and every choice always leads to same display.

\begin{tabular}{|c|c|c|}
\hline \multicolumn{3}{|c|}{ "Display } \\
\hline \multicolumn{2}{|c|}{$\begin{array}{l}\text { [R00641-0257 } \\
\text { SMITH W }\end{array}$} & $\begin{array}{r}\text { TYPE NUMERALS ONLY } \\
60 \mathrm{M} 9999999\end{array}$ \\
\hline FOR] & [YEARS & \\
\hline FOR] & [MONTHS & \\
\hline FOR] & [WEEKS & \\
\hline FOR] & [DAYS & \\
\hline FOR] & [HOURS & \\
\hline FOR] & [MINUTES & \\
\hline FOR] & [SECONDS & \\
\hline
\end{tabular}

These displays are used to enter variable numeric data. By a simple user action a cursor light spot on the screen is moved inside the bracket area; typing the appropriate number on the keyboard enters this number inside the brackets. The text with the number typed in is stored in the patient's record after pressing the end of text and transmit key. In the display shown above, each line is treated as a separate choice, the selection of which is made by typing in the numerical value required. On each of the foregoing displays the first three lines are reserved for (1) display number and instructions to the user, (2) patient identity details, and (3) the first 20 characters of the preceding choice.

\section{Free Text}

Though the aim of this record-generating system is to obtain the clinical notes as a set of assembled units of predetermined text, it seemed essential to offer the doctor the capacity to add free narrative at almost any point of the record. The user does this in one of two ways. (1) By selecting a choice from a screen by typing the choice number, following this by a special character, and then the free text. This text appears at the top of the screen as it is typed. When it is transmitted it is stored after the fixed text of the choice as words within round brackets. (2) If the user wishes to add free text without reference to any particular choice he types an $\mathrm{N}$ on the keyboard. This provides a clear screen and again the message is typed on to it. This appears in the patient's medical record file between inverted commas. Up to 120 characters may be entered in this way. 


\section{Message Verification and "Holds"}

Operationally the whole display system is an interlocked multirooted decision tree. This is shown schematically in part in Fig. 2. As the pieces of text are strung together they constitute, at certain points, a syntactically complete structure. These points of completion are built into the structure of the computer-held display system and automatically terminate each "measage." When one of these terminations is reached the whole message starting from the first text after preceding termination is displayed on the visual display unit screen as follows.

\author{
MESSAGE VERIFICATION \\ 60 M 9999999 \\ 7-FOLLOW UP NOTES \\ *ABDOMFN : MARKED TENDERNESS - RIGHT -
SUPRAPUBIC REGION - GUARDING - LOWER AB \\ DOMEN *MASS PALPABLE : EDGE WELL DEFINE
D - TENDER - FIXED - HARD - DOES NOT MOV \\ E WITH RESPIRATION - 5. CMS - BY 5 . C \\ MS \\ GIVE RATING FACTOR IF OK \\ OTHERWISE $B=B A C K ~ R=$ RESTART $L=$ LOGOUT
}

In this screen the punctuation is determined by the type of display from which the words were taken and no effort is made to provide a correct format. This enables the doctor to verify this segment of his notes. If he is satisifed with this he is able to transmit this "message" to the patient's record. If he wishes to amend what has been "written" he can, by typing B on the keyboard, backtrack through the displays and amend as necessary. When a verification takes place the computer programme causes a jump back up the display tree to some display on the branch of this tree from which the last message was constructed. This display is then shown on the screen. The displays to which this recoil action occurs are said to be "held," and this feature, like word suppression and termination, is determined by the display designer. The "hold" also has the effect of preserving all the unsuppressed text on the display tree above the hold (Fig. 2).

\section{Function Codes}

At almost any point in the course of entering the clinical notes the doctor can take certain actions by typing in a specified single character. These characters are known as "function codes." A list of some of these and their uses is given below :

$E$ : This character causes a termination to take place at the point of its use and the assembled message is then presented in the same way as is shown for automatic termination.

$B$ : This character causes a jump back to the previous display shown.

$Y$ : This causes a jump back up the display tree as far as the next display on which a "hold" exists.

$X$ : This causes a jump to the top of the whole display tree

$N$ : This will produce an empty screen on which can be typed up to 3 lines ( 120 characters) of free narrative text.

$L$ : This produces a log-out of the user and therefore is used to terminate the doctor's recording session.

\section{Delete Capacity}

Except when registration of a patient is taking place the King's College Hospital system has been designed to prevent any deletion of records that have been filed after verification. Up until this time it is possible to backtrack, using the $B$ function code to alter choice or amend text. This design feature was deliberately built into the computer programmes because of the serious medicolegal implications which could follow from allowing deletion of records.

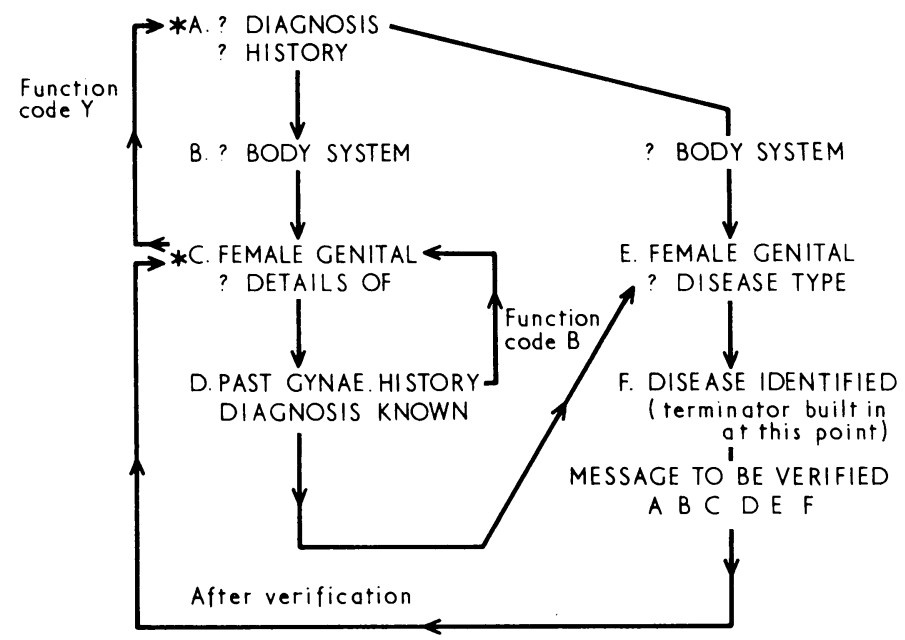

FIG. 2.-This shows, schematically, some of the interconnexions between displays, verification, and function codes. For reasons of clarity actual display text is not shown; instead the displays are presented as questions. A message construction follows the arrows. The letters adjacent to the displays represent a line of text chosen. The displays with "holds" are marked with an asterisk. Any further message starting at $\mathrm{C}$ will always have text $A$ and $B$ at the head of the message.

\section{Gaining Access to the Computer}

To use the computer system the doctor finds a visual display unit which is not in use, and by typing in his personal code gains access to the medical record file. At this point he may choose to enter notes about a previously registered patient, register a new patient, or inspect an existing record. If he chooses to register a patient an appropriate " $R$ " type display is presented and the required details can be entered.

If he wishes to record "clinical notes" the doctor takes this numbered choice and is presented with a display which requests the patient's number. This is entered within the brackets and appropriate keyboard action causes the computer programme to identify this number. If this patient's details already exist on the disc file they are displayed for verification. If, by some error, no patient registration number corresponding to the entered number has been filed an "empty" registration display is presented with a comment above the fixed text indicating that this number represents a new patient for whom no computer record exists.

Every user is allocated a personal code, which acts not only as the entry to the computer system but represents his personal "signature" to all notes which he makes about patients. The date and time of all messages recorded are also kept automatically by the computer with the text of the message.

\section{Printed Record}

At a particular time during each day, determined essentially by the computer staff, special computer programmes are run which have the effect of "tidying" the magnetic record and producing a printed record for each patient. This printed record has a format which is determined by a programme that interprets the magnetically held record. The patient data are presented under the primary headings of history, physical examination, etc. A typical example of this format is presented in Fig. 3.

The printed records are assembled and dispatched to the ward during the late afternoon. Any previously existing printed records are destroyed and the new version is substituted in their place in the patient's note folder. In addition to this printed record, a special nursing activities list is printed by the computer once a day. This list contains an organized schedule of nursing activities obtained from the basic nursing treatment orders previously entered in the system by the ward sister. 


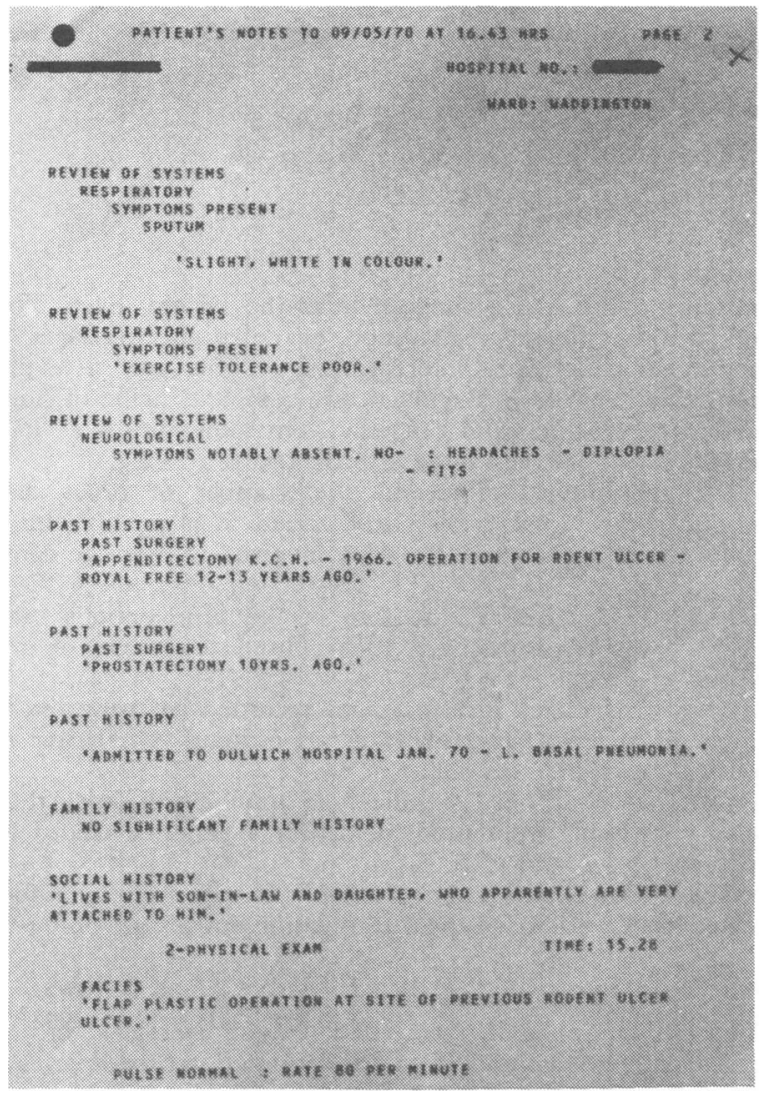

FIG. 3.-Typical example of the printed format.

One of the reasons for producing a printed record is so that in the event of any major computer breakdown a set of notes current up to the previous 24-hour print out is always available. In addition, interim print outs are produced two or three times daily, but these are not distributed to the wards unless a breakdown occurs.

\section{Archiving}

When a patient is discharged the computer programme causes the medical record to be erased from the exchangeable disc and copied on to an archive magnetic tape. The final version of the printed record is also produced on this occasion and is dispatched to the ward, where it follows the same course as the traditional handwritten notes, becoming the source of a medical summary letter and ultimately being filed on the medical folder in the hospital records department.

\section{Implementation}

We conclude this account with a description of the time required to bring phase 1 into operation and very rough estimates of human resources to achieve the working system. Work on the project started formally in January 1968, though much of the first nine months was devoted to problems which have had little subsequent relevance to phase 1 project to date. The computer (ICL 1905 E) was delivered in April 1969 and phase 1 system went "live" in the wards early in February 1970.

The training schedule for medical and nursing staff who were to use the system was limited to a short lecture course. Additionally, there were demonstrations for consultants and registrars and about eight hours of more intensive system use and training for junior house staff. Some training was also given to ward clerks, secretaries, and medical students. The responsibility for training house staff was given to a systems analyst who was not medically qualified.

It is difficult to measure the human resources used to bring phase $1 \mathrm{KCH}$ project to fruition. The programming itself represented about nine man-years, while display development and design probably accounted for about five man-years.

\section{Discussion}

Even given the limitations imposed by equipment, staff, and time, it must not be assumed that the system adopted at King's College Hospital is the best available, and a series of discrete modifications and extensions are planned for the near future. Any realistic assessment of the value which such a computer system might have to the clinician will always depend on the acceptance or otherwise of the system by the user at ward level.

This paper contains no presentation or discussion of the objectives of the project, nor can it attempt any assessment of the value of such projects in general. In a further paper we review the use of this experimental system with ward-based terminals as means of storing and retrieving the clinical record (Opit and Woodroffe, 1970).

This project is one of a number financed by the Department of Health and is under the medical direction of Professor J. Anderson.

REFERENCE

Opit, L. J., and Woodroffe, F. J. (1970) .British Medical fournal, 4, 80. 